\title{
Association of the serum uric acid level with liver histology in biopsy-proven non-alcoholic fatty liver disease
}

\author{
QIAN HUANG ${ }^{1}$, JIANHUA YU ${ }^{2}$, XIANTU ZHANG ${ }^{3}$, SHOURONG LIU $^{4}$ and YANYAN GE ${ }^{5}$ \\ Departments of ${ }^{1}$ Hepatology, ${ }^{2}$ Infectious Disease and ${ }^{3}$ Pathology; ${ }^{4}$ Institute of Liver Disease, Hangzhou Xixi Hospital \\ Affiliated to Zhejiang Chinese Medical University, Hangzhou, Zhejiang 310023; ${ }^{5}$ Department of Internal Medicine, The Third \\ People's Hospital of Hangzhou Affiliated to Zhejiang Chinese Medical University, Hangzhou, Zhejiang 310009, P.R. China
}

Received February 15, 2016; Accepted April 27, 2016

DOI: $10.3892 /$ br.2016.698

\begin{abstract}
Hyperuricemia is significantly associated with and independently predicts the risk for non-alcoholic fatty liver disease (NAFLD). The aim of the present study was to examine the association of serum uric acid (SUA) levels with liver histology in patients with biopsy-proven NAFLD. Data were collected from 158 adults aged $>18$ years, and diagnosed with biopsy-proven NAFLD. The differences in liver histology were assessed between hyperuricemic and normal SUA groups with NAFLD to determine the possible risk factors. The SUA level was closely associated with the degree of steatosis (correlation coefficient 0.177, $\mathrm{P}=0.027$ ). A higher proportion of patients with hyperuricemia showed increased severity of lobular inflammation (lobular inflammation score $\geq 2$ ) compared with patients exhibiting normal SUA (75 vs. $52.7 \% ; \chi^{2}=8.548, \mathrm{P}=0.003$ ). Hyperuricemic groups had higher non-alcoholic steatosis $(\geq 5)$ compared to the normal SUA groups with NAFLD (48.8 vs. $\left.31.1 \% ; \chi^{2}=5.131, \mathrm{P}=0.024\right)$. Hyperuricemia was independently associated with advanced lobular inflammation (odds ratio, 2.79; 95\% confidence interval, 1.250-6.257; $\mathrm{P}=0.012$ ) using a logistic regression model controlling for ferritin, serum alanine aminotransferase and aspartate aminotransferase. In conclusion, hyperuricemia is associated with histologically severe NAFLD. Hyperuricemia was independently associated with greater odds of advanced lobular inflammation of NAFLD.
\end{abstract}

Correspondence to: Dr Qian Huang, Department of Hepatology, Hangzhou Xixi Hospital Affiliated to Zhejiang Chinese Medical University, 2 Hengbu Street, Hangzhou, Zhejiang 310023, P.R. China E-mail: tcm99hq@163.com

Abbreviations: NAFLD, non-alcoholic fatty liver disease; SUA, serum uric acid; NASH, non-alcoholic steatohepatitis; BMI, body mass index; ALT, alanine aminotransferase; AST, aspartate aminotransferase; GGT, $\gamma$-glutamyl transpeptidase

Key words: histology, inflammation, non-alcoholic fatty liver disease, uric acid

\section{Introduction}

Non-alcoholic fatty liver disease (NAFLD) is a state of intrahepatic fat accumulation, ranging from simple steatosis to non-alcoholic steatohepatitis (NASH) and cirrhosis. NAFLD is the most common liver disease in Western countries, affecting 20-30\% of the general population (1). NAFLD is also an emerging public health concern in developing countries $(2,3)$. Among more affluent regions of China, the community prevalence of NAFLD is $15 \%$ (2). With the increasing obesity pandemic, the prevalence of NAFLD is likely to increase in the future (2). Serum uric acid (SUA) levels are closely associated with insulin resistance, diabetes mellitus type 2 and metabolic syndrome $(4,5)$. Hyperuricemia was significantly associated with NAFLD, and the prevalence of NAFLD increases with SUA increase (6-8). Elevation in SUA levels independently predicts an increase in the risk for the incident of NAFLD and suggests that high SUA levels may have a causal role in the development of NAFLD (9). Certain in vitro and in vivo studies in hepatocytes and liver tissue of mice suggest that uric acid stimulated fat synthesis and induced inflammatory cell infiltration in the liver $(10,11)$. Therefore, it is plausible that uric acid may reflect increased disease severity in NAFLD. However, there is one previous study regarding the association between UA serum levels and histological severity of NAFLD patients (12). The majority of studies have been conducted in Western populations. Notably, the prevalence of obesity in China is significantly lower compared to Western countries $(13,14)$, and certain features of metabolic syndrome, such as waist circumference and body mass index (BMI), are defined differently $(2,15)$. Therefore, due to this the previous results should not be compared to the Chinese population. The present study aimed to investigate the association of SUA levels with liver histology in Chinese patients with biopsy-proven NAFLD.

\section{Materials and methods}

Study design and subjects

Patient population. A total of 158 patients (age $\geq 18$ years) with well-characterized and liver biopsy-confirmed NAFLD were included in the study. Patients were untreated and consecutively biopsied at the Hangzhou Xixi Hospital Affiliated to 
Zhejiang Chinese Medical University (Hangzhou, Zhejiang, China) between 2008 and 2013. The diagnosis of NAFLD was based on the following criteria: Liver biopsy showing steatosis in $\geq 5 \%$ of hepatocytes. The exclusion criteria were as follows: Viral hepatitis or autoimmune, alcohol-induced (male $>20 \mathrm{~g} /$ day; female $>10 \mathrm{~g} /$ day) or drug-induced liver disease, and cholestatic or metabolic/genetic liver disease. Other liver diseases were excluded using specific clinical, biochemical, radiographic and/or histological criteria.

Clinical and laboratory assessments. Clinical and laboratory parameters were collected on the day of the liver biopsy. A complete medical history and physical examination was undertaken in all patients. Hyperuricemia was defined as an SUA level $>360 \mathrm{mmol} / 1$ in women and $>420 \mathrm{mmol} / 1$ in men (16). BMI was calculated as weight (in kilograms)/height (in meters ${ }^{2}$ ). A fasting blood sample was obtained from each subject in the morning. Laboratory measurements included serum alanine aminotransferase (ALT) and aspartate aminotransferase (AST), total bilirubin, alkaline phosphatase, $\gamma$-glutamyl transpeptidase (GGT) and albumin levels; fasting glucose, complete blood count; total cholesterol, high-density lipoprotein cholesterol (HDL-C), low-density lipoprotein cholesterol (LDL-C) and total triglycerides; fasting insulin; ferritin levels; viral serology for hepatitis B and C infection; autoantibodies; $\alpha 1$ antitrypsin levels and phenotype. All the values were measured using an Olympus AU640 autoanalyzer (Olympus, Kobe, Japan) adopting the standard methods.

For histological analysis, liver specimens were fixed with $4 \%$ buffered paraformaldehyde and embedded in paraffin. Sections of $2 \mu \mathrm{m}$ were prepared and evaluated for liver fibrosis by hematoxylin and eosin staining. The histological grading and staging of NAFLD (degree of steatosis, lobular inflammation and hepatocyte ballooning) were scored according to the modified criteria established by Kleiner et al (17). The degree of steatosis was assessed using the following scale: 0 (absent, when the extent of steatosis was <5\%), 1 (5-33\%), 2 (33-66\%), and $3(>66 \%)$. The NAFLD activity score (NAS) ranges between 0 and 8 , and is represented by the sum of scores for steatosis (0-3), lobular inflammation (0-3) and ballooning (0-2). NAS $\geq 5$ were diagnosed as NASH. Degree of fibrosis was evaluated separately according to the Kleiner and Brunt score (17). Fibrosis was staged as: 0 (none), 1 (portal or zone 3 perisinusoidal fibrosis), 2 (periportal and zone 3 perisinusoidal fibrosis without bridging), 3 (bridging fibrosis) and 4 (cirrhosis). Liver biopsy specimens were analyzed by an expert liver pathologist who was blinded to the biological data and order of biopsy.

Statistical analyses. Continuous variables are presented as mean \pm standard deviation. The association between SUA and variables of interest was examined with uric acid as a dichotomous variable. Continuous variables were compared with Student's t-test or Wilcoxon rank-sum test, depending on the normality of the data. The association between SUA level and histology was determined using Spearman's rank correlation. Categorical variables, including histological features such as steatosis, lobular inflammation grade and fibrosis stage, were analyzed using either Fisher's exact or $\chi^{2}$ tests. Binary logistic regression analysis (enter; entry: 0.05, removal: 0.10) was used to evaluate the risk factors for NAFLD. All the statistical analyses were performed using the SPSS software package version 20 for Windows (IBM, Corp., Armonk, NY, USA). $\mathrm{P}<0.05$ (two-tailed) was considered to indicate a statistically significant difference.

\section{Results}

Patient profile. A total of 158 adult subjects (aged $\geq 18$ years) with biopsy-proven NAFLD (defined as $>5 \%$ steatosis) and SUA levels measured within 2 weeks of liver biopsy, were evaluated. The baseline profile, including age, gender, BMI, blood pressure, serum liver enzymes, lipids, glucose and SUA levels, are shown in Table I. Hyperuricemia was identified in 84 subjects ( 73 males and 11 females). The proportion of normal SUA and hyperuricemia was 46.8 vs. $53.2 \%$, respectively. No other significant differences were observed in SBP/DBP or $\mathrm{BMI}$ and the presence of diabetes between normal SUA and hyperuricemia groups.

Association between the serum parameters and SUA levels. Differences in the serum parameters between patients with normal SUA versus hyperuricemia are shown in Table I. Patients with hyperuricemia showed higher hemoglobin and LDL-C, but lower fasting glucose compared to those with normal uric acid levels $(\mathrm{P}=0.016, \mathrm{P}=0.028$ and $\mathrm{P}=0.038$, respectively). By contrast, no difference was observed between the groups for GGT, direct bilirubin, fasting insulin, homeostasis model assessment of insulin resistance, white blood cell count and platelet count. Although AST, ALT and serum ferritin in the hyperuricemia group were higher compared to the normal uric acid group, no significance was detected $(\mathrm{P}=0.209, \mathrm{P}=0.089$ and $\mathrm{P}=0.335$, respectively).

Association between SUA and histological severity of NAFLD. First, the association of the SUA level and liver histology of all the NAFLD patients was examined. The results showed that the SUA level was closely associated with the degree of steatosis $(\mathrm{P}=0.027)$ in all patients. The SUA level was possibly associated with NAS and lobular inflammation $(\mathrm{P}=0.056$, $\mathrm{P}=0.059)$, but not with the degree of hepatocyte ballooning and fibrosis $(\mathrm{P}=0.689, \mathrm{P}=0.584)$.

Increased histological severity of NAFLD was associated with hyperuricemia. A higher proportion of patients with hyperuricemia showed increased severity of lobular inflammation compared with patients exhibiting normal uric acid levels $\left(\chi^{2}=8.548, \mathrm{P}=0.003\right)$ (Fig. 1). Subjects with hyperuricemia had a higher NAS (NAS $\geq 5$ ) compared to the normal SUA groups with NAFLD $\left(\chi^{2}=5.131, \mathrm{P}=0.024\right)$ (Fig. 2). Subjects with hyperuricemia were also likely to have a definitive diagnosis of NASH compared to those with normal SUA. However, no significant differences occurred between hyperuricemia and normal SUA groups in terms of the proportion of patients with fibrosis, ballooning and steatosis $(\mathrm{P}=0.249, \mathrm{P}=0.653$ and $\mathrm{P}=0.247$, respectively).

To investigate the different levels of SUA independently associated with lobular inflammation and NASH, we used a binary logistic regression analysis, including the following variables, selected a priori, AST, ALT, ferritin and HDL-C, and SUA values represented dichotomous independent variables (normal SUA and hyperuricemia). These results showed 
Table I. Characteristics of patients according to different serum uric acid levels.

\begin{tabular}{|c|c|c|c|c|}
\hline Variable & Normal SUA & Hyperuricemia & $\mathrm{t}$ value & P-value \\
\hline Age, year & $38.52 \pm 10.62$ & $34.72 \pm 9.81$ & 2.338 & 0.021 \\
\hline Total, n (male/female) & $74(54 / 20)$ & $84(73 / 11)$ & 4.842 & $0.028^{\mathrm{a}}$ \\
\hline $\mathrm{BMI}, \mathrm{kg} / \mathrm{m}^{2}$ & $25.79 \pm 2.53$ & $25.68 \pm 4.60$ & 0.180 & 0.858 \\
\hline $\mathrm{SBP}, \mathrm{mmHg}$ & $121.86 \pm 11.44$ & $123.48 \pm 14.01$ & -0.698 & 0.486 \\
\hline $\mathrm{DBP}, \mathrm{mmHg}$ & $79.06 \pm 7.42$ & $77.95 \pm 10.55$ & 0.672 & 0.503 \\
\hline Diabetes diagnosis, $\mathrm{n}$ & 3 & 2 & 0.021 & $0.885^{\mathrm{a}}$ \\
\hline AST, IU/1 & $58.50 \pm 49.58$ & $68.26 \pm 47.62$ & -1.261 & 0.209 \\
\hline ALT, IU/1 & $92.32 \pm 94.42$ & $116.77 \pm 84.94$ & -1.713 & 0.089 \\
\hline WBC, $\times 10^{9} / 1$ & $6.39 \pm 1.74$ & $6.69 \pm 1.65$ & -1.071 & 0.286 \\
\hline PLT, $x 10^{9} / 1$ & $196.39 \pm 55.42$ & $203.40 \pm 56.57$ & -0.785 & 0.434 \\
\hline $\mathrm{HB}, \mathrm{g} / \mathrm{l}$ & $14.55 \pm 2.00$ & $15.24 \pm 1.16$ & -2.439 & 0.016 \\
\hline GGT, IU/1 & $88.47 \pm 93.78$ & $84.94 \pm 69.02$ & 0.271 & 0.786 \\
\hline Glucose, mmol/1 & $5.52 \pm 1.46$ & $5.13 \pm 0.75$ & 2.090 & 0.038 \\
\hline Insulin, mIU/l & $11.73 \pm 7.00$ & $14.06 \pm 7.40$ & -1.788 & 0.076 \\
\hline $\mathrm{TG}, \mathrm{mmol} / \mathrm{l}$ & $2.16 \pm 1.31$ & $2.18 \pm 1.79$ & -0.093 & 0.926 \\
\hline $\mathrm{TC}, \mathrm{mmol} / \mathrm{l}$ & $4.92 \pm 1.23$ & $5.13 \pm 0.99$ & -1.159 & 0.248 \\
\hline HDL-C, mmol/1 & $1.15 \pm 0.26$ & $1.20 \pm 0.34$ & -0.962 & 0.338 \\
\hline LDL-C, mmol/l & $2.58 \pm 0.92$ & $2.96 \pm 0.88$ & -2.227 & 0.028 \\
\hline Ferritin, ng/ml & $359.21 \pm 267.99$ & $404.27 \pm 248.11$ & -0.967 & 0.335 \\
\hline Uric acid, $\mu \mathrm{mol} / 1$ & $346.10 \pm 53.45$ & $471.13 \pm 50.25$ & -15.101 & $<0.0001$ \\
\hline
\end{tabular}

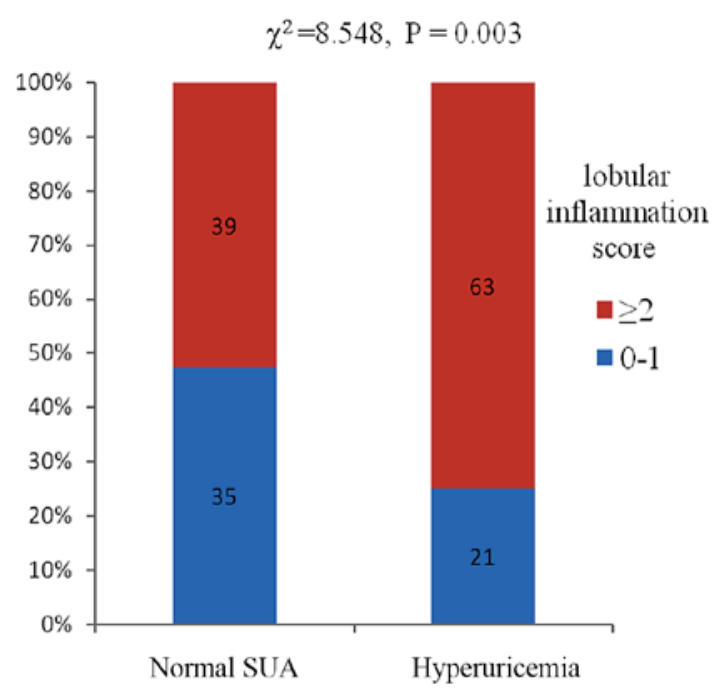

Figure 1. Association between different SUA and the severity of lobular inflammation with non-alcoholic fatty liver disease. SUA, serum uric acid.

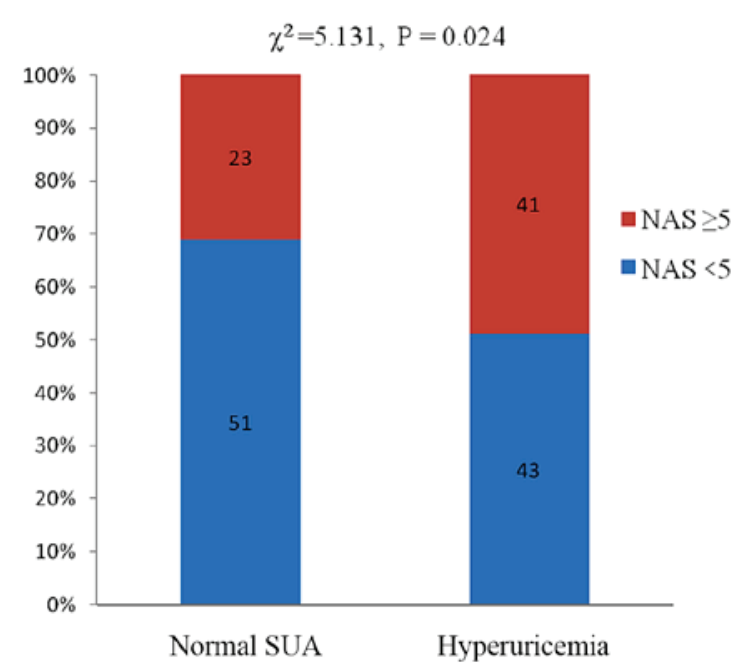

Figure 2. Association between different SUA and NAS with NAFLD. NAFLD, non-alcoholic fatty liver disease; SUA, serum uric acid; NAS, NAFLD activity score. that hyperuricemia was an independent predictor of advanced lobular inflammation [odds ratio (OR), 2.797; 95\% confidence interval (CI), 1.250-6.257; $\mathrm{P}=0.012$ ] using a logistic regression model controlled for ferritin, serum ALT and AST (Table II). Hyperuricemia was a more likely predictor of NASH diagnosis (OR, 2.28; $\mathrm{P}=0.052)$.

\section{Discussion}

In the present study, the SUA level was significantly associated with the degree of steatosis in patients with NAFLD. Furthermore, increased SUA levels were associated with a greater severity of lobular inflammation and NASH. 
Table II. Independent association of advanced lobular inflammation on logistic regression modeling using different serum uric acid levels.

\begin{tabular}{lcccccc}
\hline Variable & $\mathrm{B}$ & $\mathrm{SE}$ & Wald & P-value & Exp(B) & 95\% CI for Exp(B) \\
\hline Uric acid & 1.028 & 0.411 & 6.265 & 0.012 & 2.797 & $1.250-6.257$ \\
AST & 0.009 & 0.009 & 0.937 & 0.333 & 1.009 & $0.991-1.026$ \\
ALT & 0.000 & 0.005 & 0.002 & 0.961 & 1.000 & $0.991-1.009$ \\
Ferritin & 0.001 & 0.001 & 1.890 & 0.169 & 1.001 & $0.999-1.003$ \\
\hline
\end{tabular}

SE, standard error; CI, confidence interval; AST, aspartate aminotransferase; ALT, alanine aminotransferase.

Hyperuricemia was independently associated with an increased risk of severity of lobular inflammation and was a likely predictor of NASH in NAFLD patients.

SUA levels were significantly associated with the degree of steatosis in patients with NAFLD. Uric acid has been shown to contribute to lipoprotein oxidation and inflammation $(11,18)$. Lanaspa et al (10) provided the first direct evidence that uric acid stimulated fat synthesis in the hepatocytes and in the pound mouse. The study reported that lowering the hepatic uric acid level reduced fatty liver in the fructose-exposed hepatocytes (10). Another study also indicated that hypouricemic therapy using allopurinol and benzbromarone significantly ameliorated hepatic steatosis and decreased serum cholesterol levels in a Mongolian gerbil model of NAFLD (19). In fact, a population-based prospective study showed that SUA was significantly associated with the development of NAFLD, suggesting that high SUA levels may have a causal role in the development of NAFLD (9). Similarly, the present study demonstrated that the SUA level was significantly associated with the degree of steatosis from a histological perspective.

As aforementioned, the present study identified that increased SUA levels were associated with a greater severity of lobular inflammation in biopsy-proven NAFLD. Another study reported similar findings in Italy (12). The present study observed that hyperuricemia was also possibly linked to higher NAS, implying that hyperuricemia was likely to be associated with a definitive diagnosis of NASH. This result further demonstrated that hyperuricemia was an independent predictor of advanced lobular inflammation (OR, 2.797; 95\% CI, 1.250-6.257; $\mathrm{P}=0.012$ ) using a logistic regression modeling.

Three potential factors explain the association between high SUA levels and severity of lobular inflammation. First, uric acid acts as a strong oxidant. Uric acid activated NADPH oxidase followed by redox-dependent proinflammatory signaling via protein kinase p38 in adipocyte differentiation (18). Soluble uric acid directly stimulated the redox-dependent proinflammatory signaling in adipocytes. Second, SUA induced inflammatory cell infiltration in the liver. Uric acid increased the expression of MCP-1 (an inflammatory agent), and decreased the expression of adiponectin (an anti-inflammatory agent). Third, lowering uric acid in these mice by inhibiting xanthine oxidoreductase improved the balance in the adipose tissue by attenuating MCP-1 levels and increasing the production of adiponectin. In addition, lowering uric acid in obese mice decreased macrophage infiltration in the adipose tissue and reduced insulin resistance. Hyperuricemia may be partially responsible for the inflammation in the adipose tissue (11). A recent study demonstrated that uric acid crystals induced pro-inflammatory effects (20).

NLRP3 inflammasomes have a pivotal role in triggering innate immune responses in NAFLD. Uric acid crystals activate the NALP3 inflammasome (21). Uric acid is a classic example of the danger-associated molecular pattern. Vandanmagsar et al (22) found that the NALP3 inflammasome mediated the recognition of certain non-microbial 'danger-signals' leading to caspase-1 activation and interleukin-1 $\beta$ (IL-1 $\beta$ ) and IL-18 secretion, and contributed to obesity-induced inflammation and insulin resistance. Elimination of NALP3 in obesity reduced IL-18 and adipose tissue interferon- $\gamma$ along with an increase in naïve $\mathrm{T}$ cells and reduction in effector adipose tissue $\mathrm{T}$ cells. Uric acid stimulated NLRP3 inflammasomes to produce IL-1 $\beta$ in macrophages and promoted chemokine signaling (23). A recent study showed that uric acid was increased in the serum and liver of alcohol-fed mice. Depletion of uric acid with allopurinol provided significant protection from alcohol-induced inflammatory response, steatosis and liver damage (24). Other clinical studies have also demonstrated that lowering uric acid benefited inflammation, endothelial function, blood pressure and insulin resistance (25-27). These studies demonstrated that uric acid induced an inflammatory response and inflammatory cell infiltration in numerous ways. The study by Petta et al (12) in Italy showed similar results, indicating that hyperuricemia was independently associated with lobular inflammation. Despite a small sample size, the present study was similar to that of Petta et al (12), representing different ethnicities with similar findings. These results indicated that hyperuricemia has an important role in inducing inflammation. A cross-sectional study, such as the present study, cannot definitively confirm whether hyperuricemia was a cause or a marker of hepatic necroinflammation. However, it was a valuable marker of hepatic necrosis.

In a clinical cohort study, Afzali et al (28) found that the SUA level was associated with the development of cirrhosis. However, the present study showed that hyperuricemia was not associated with advanced fibrosis in NAFLD; the study populations were different. The present subjects were patients with biopsy-proven NAFLD in China. The patients in the study by Afzali et al included different ethnicities and liver diseases, with varied etiopathogeneses. Hyperuricemia is significantly associated with NAFLD (6-8). However, SUA was normal in 
a number of patients with NAFLD, which indicated that SUA mediated the pathogenesis of NAFLD, but in conjunction with other factors. The present results revealed no association between hyperuricemia and advanced fibrosis of NAFLD. However, SUA levels were associated with greater severity of lobular inflammation and NASH. Others studies demonstrated that uric acid induced inflammation and inflammatory cell infiltration in a variety of ways (25-27). Repeated inflammation leads to liver fibrosis suggesting that hyperuricemia was indirectly involved in liver fibrosis. The present cross-sectional study did not confirm the causal association between hyperuricemia and liver fibrosis. Additional prospective studies are required to provide evidence linking hyperuricemia with liver fibrosis.

The limitations of the present study are associated with the cross-sectional design, which cannot determine any causality between uric acid levels and NAFLD histology. In addition, the interpretation of the results may be affected by the small sample size. Further studies are required to reveal the detailed association and possible mechanisms underlying the association between SUA and NAFLD.

In conclusion, the SUA level is significantly associated with the degree of steatosis. Hyperuricemia is associated with a greater severity of lobular inflammation and higher NAS. Hyperuricemia was independently associated with greater odds of advanced lobular inflammation of NAFLD. In the future, large-scale studies are required to develop novel therapeutic targets and intervention strategies for NAFLD.

\section{References}

1. Jimba S, Nakagami T, Takahashi M, Wakamatsu T, Hirota Y, Iwamoto Y and Wasada T: Prevalence of non-alcoholic fatty liver disease and its association with impaired glucose metabolism in Japanese adults. Diabet Med 22: 1141-1145, 2005.

2. Fan JG and Farrell GC: Epidemiology of non-alcoholic fatty liver disease in China. J Hepatol 50: 204-210, 2009.

3. Amarapurkar DN, Hashimoto E, Lesmana LA, Sollano JD, Chen PJ and Goh KL; Asia-Pacific Working Party on NAFLD: How common is non-alcoholic fatty liver disease in the Asia-Pacific region and are there local differences? J Gastroenterol Hepatol 22: 788-793, 2007.

4. Hjortnaes J, Algra A, Olijhoek J, Huisman M, Jacobs J, van der Graaf Y and Visseren F: Serum uric acid levels and risk for vascular diseases in patients with metabolic syndrome. J Rheumatol 34: 1882-1887, 2007.

5. Choi HK and Ford ES: Prevalence of the metabolic syndrome in individuals with hyperuricemia. Am J Med 120: 442-447, 2007.

6. Li Y, Xu C, Yu C, Xu L and Miao M: Association of serum uric acid level with non-alcoholic fatty liver disease: A cross-sectional study. J Hepatol 50: 1029-1034, 2009.

7. Sirota JC, McFann K, Targher G, Johnson RJ, Chonchol M and Jalal DI: Elevated serum uric acid levels are associated with non-alcoholic fatty liver disease independently of metabolic syndrome features in the United States: Liver ultrasound data from the National Health and Nutrition Examination Survey. Metabolism 62: 392-399, 2013.

8. Lee YJ, Lee HR, Lee JH, Shin YH and Shim JY: Association between serum uric acid and non-alcoholic fatty liver disease in Korean adults. Clin Chem Lab Med 48: 175-180, 2010.

9. Xu C, Yu C, Xu L, Miao M and Li Y: High serum uric acid increases the risk for nonalcoholic Fatty liver disease: A prospective observational study. PLoS One 5: e11578, 2010.

10. Lanaspa MA, Sanchez-Lozada LG, Choi YJ, Cicerchi C, Kanbay M, Roncal-Jimenez CA, Ishimoto T, Li N, Marek G, Duranay M, et al: Uric acid induces hepatic steatosis by generation of mitochondrial oxidative stress: Potential role in fructose-dependent and -independent fatty liver. J Biol Chem 287: 40732-40744, 2012.
11. Baldwin W, McRae S, Marek G, Wymer D, Pannu V, Baylis C, Johnson RJ and Sautin YY: Hyperuricemia as a mediator of the proinflammatory endocrine imbalance in the adipose tissue in a murine model of the metabolic syndrome. Diabetes 60: 1258-1269, 2011.

12. Petta $\mathrm{S}$, Cammà $\mathrm{C}$, Cabibi $\mathrm{D}, \mathrm{Di}$ Marco $\mathrm{V}$ and Craxì $\mathrm{A}$ : Hyperuricemia is associated with histological liver damage in patients with non-alcoholic fatty liver disease. Aliment Pharmacol Ther 34: 757-766, 2011

13. Flegal KM, Carroll MD, Kit BK and Ogden CL: Prevalence of obesity and trends in the distribution of body mass index among US adults, 1999-2010. JAMA 307: 491-497, 2012.

14. Reynolds K, Gu D, Whelton PK, Wu X, Duan X, Mo J and He J; InterASIA Collaborative Group: Prevalence and risk factors of overweight and obesity in China. Obesity (Silver Spring) 15: 10-18, 2007.

15. Grundy SM, Brewer HB Jr, Cleeman JI, Smith SC Jr and Lenfant C; American Heart Association, National Heart, Lung, and Blood Institute: Definition of metabolic syndrome: Report of the National Heart, Lung, and Blood Institute/American Heart Association conference on scientific issues related to definition. Circulation 109: 433-438, 2004.

16. Fang $\mathrm{J}$ and Alderman MH: Serum uric acid and cardiovascular mortality the NHANES I epidemiologic follow-up study, 1971-1992. National Health and Nutrition Examination Survey. JAMA 283: 2404-2410, 2000.

17. Kleiner DE, Brunt EM, Van Natta M, Behling C, Contos MJ, Cummings OW, Ferrell LD, Liu YC, Torbenson MS, Unalp-Arida A, et al; Nonalcoholic Steatohepatitis Clinical Research Network: Design and validation of a histological scoring system for nonalcoholic fatty liver disease. Hepatology 41: 1313-1321, 2005

18. Sautin YY, Nakagawa T, Zharikov S and Johnson RJ: Adverse effects of the classic antioxidant uric acid in adipocytes: NADPH oxidase-mediated oxidative/nitrosative stress. Am J Physiol Cell Physiol 293: C584-C596, 2007.

19. Xu CF, Yu CH, Xu L, Sa XY and Li YM: Hypouricemic therapy: A novel potential therapeutic option for nonalcoholic fatty liver disease. Hepatology 52: 1865-1866, 2010.

20. Crane JK and Mongiardo KM: Pro-inflammatory effects of uric acid in the gastrointestinal tract. Immunol Invest 43: 255-266, 2014.

21. Martinon F: Signaling by ROS drives inflammasome activation. Eur J Immunol 40: 616-619, 2010.

22. Vandanmagsar B, Youm YH, Ravussin A, Galgani JE, Stadler K, Mynatt RL, Ravussin E, Stephens JM and Dixit VD: The NLRP3 inflammasome instigates obesity-induced inflammation and insulin resistance. Nat Med 17: 179-188, 2011.

23. Kim SM, Lee SH, Kim YG, Kim SY, Seo JW, Choi YW, Kim DJ, Jeong KH, Lee TW, Ihm CG, et al: Hyperuricemia-induced NLRP3 activation of macrophages contributes to the progression of diabetic nephropathy. Am J Physiol Renal Physiol 308: F993-F1003, 2015

24. Iracheta-Vellve A, Petrasek J, Satishchandran A, Gyongyosi B, Saha B, Kodys K, Fitzgerald KA, Kurt-Jones EA and Szabo G: Inhibition of sterile danger signals, uric acid and ATP, prevents inflammasome activation and protects from alcoholic steatohepatitis in mice. J Hepatol 63: 1147-1155, 2015.

25. Kanbay M, Huddam B, Azak A, Solak Y, Kadioglu GK, Kirbas I, Duranay M, Covic A and Johnson RJ: A randomized study of allopurinol on endothelial function and estimated glomular filtration rate in asymptomatic hyperuricemic subjects with normal renal function. Clin J Am Soc Nephrol 6: 1887-1894, 2011.

26. Ogino $\mathrm{K}$, Kato $\mathrm{M}$, Furuse $\mathrm{Y}$, Kinugasa $\mathrm{Y}$, Ishida $\mathrm{K}$, Osaki S, Kinugawa T, Igawa O, Hisatome I, Shigemasa C, et al: Uric acid-lowering treatment with benzbromarone in patients with heart failure: A double-blind placebo-controlled crossover preliminary study. Circ Heart Fail 3: 73-81, 2010.

27. Kanbay M, Ozkara A, Selcoki Y, Isik B, Turgut F, Bavbek N, Uz E, Akcay A, Yigitoglu R and Covic A: Effect of treatment of hyperuricemia with allopurinol on blood pressure, creatinine clearence, and proteinuria in patients with normal renal functions. Int Urol Nephrol 39: 1227-1233, 2007.

28. Afzali A, Weiss NS, Boyko EJ and Ioannou GN: Association between serum uric acid level and chronic liver disease in the United States. Hepatology 52: 578-589, 2010. 